\title{
AN IMPROVEMENT OF A TRANSCENDENCE MEASURE OF GALOCHKIN AND MAHLER'S $S$-NUMBERS
}

\author{
MASAAKI AMOU
}

(Received 26 February 1990; revised 28 August 1990)

Communicated by $\mathbf{J}$. H. Loxton

\begin{abstract}
We give a transcendence measure of special values of functions satisfying certain functional equations. This improves an earlier result of Galochkin, and gives a better upper bound of the type for such a number as an $S$-number in the classification of transcendental numbers by Mahler.
\end{abstract}

1991 Mathematics subject classification (Amer. Math. Soc.) 11 J 82.

\section{Introduction}

Let $K$ be an algebraic number field of finite degree. Let $f(z)$ be a function which is transcendental over $\mathbb{C}(z)$ and holomorphic in some neighborhood $U$ of the origin, and satisfies the functional equation

$$
f(T z)=\frac{A_{1}(z, f(z))}{A_{2}(z, f(z))}, T z=z^{r}(r \in \mathbb{N}, r \geq 2),
$$

where $A_{i}(z, y)=a_{i 1}(z) y+a_{i 2}(z) \in K[z, y](i=1,2)$. Suppose that the coefficients of $f(z)$ in its Taylor series expansion at the origin all lie in the field $K$.

Let $\alpha \in U$ be an algebraic number with $0<|\alpha|<1$ satisfying

$$
\operatorname{det}\left(a_{i j}\left(T^{k} \alpha\right)\right)_{i, j=1,2} \neq 0
$$

This research was partly supported by the Grant-in-Aid for Encouragement of Young Scientists (No. 63790126), the Ministry of Education, Science and Culture, Japan.

(C) 1992 Australian Mathematical Society 0263-6115/92 \$A2.00+0.00 
for any $k(k=0,1,2, \ldots)$. This condition allows us that $A_{2}\left(T^{k} \alpha, f\left(T^{k} \alpha\right)\right)$ $\neq 0$ for any $k(k=0,1,2, \ldots)$.

In the notation as above, Mahler proved in [4] that the number $f(\alpha)$ is transcendental. In [2], Galochkin considered a quantitative version of this result and gave the following transcendence measure of $f(\alpha)$ :

TheOREM (Galochkin [2]). In the notation as above, further, let $P(x) \in$ $\mathbb{Z}[z]$ be any nonzero polynomial whose degree is at most $d$ and whose height is at most $H$. Put

$$
b=\chi_{1}^{-1} \log L(\alpha), c=\log |\alpha|^{-1} \text { and } \chi_{0}=[K(\alpha): \mathbb{Q}],
$$

where $\chi_{1}$ is the degree of $\alpha$ and $L(\alpha)$ is the length of $\alpha$. Then we have

$$
|P(f(\alpha))|>H^{-(2 r+1)^{2} b c^{-1} \chi_{0}^{2} d}
$$

for all sufficiently large $H$.

Our main purpose is to sharpen this estimate. To state our results, we recall usual notation and the definition of Mahler's $S$-numbers (cf. Schneider [6]).

For any algebraic number $\alpha$ with minimal defining polynomial $Q(x)=$ $a_{0}(x-\alpha)\left(x-\alpha^{\prime}\right) \cdots\left(x-\alpha^{(x-1)}\right) \in \mathbb{Z}[x]\left(a_{0}>0\right)$, we denote by $\operatorname{den}(\alpha)$ the denominator of $\alpha$, that is, the least positive integer $d$ such that $d \alpha$ is an algebraic integer, by $\overline{|\alpha|}$ the house of $\alpha$, that is, the maximum of the absolute values of the roots of $Q(x)$, and by $M(\alpha)$ the Mahler measure of $\alpha$, that is the number which is defined by

$$
M(\alpha)=a_{0} \prod_{i=0}^{\chi-1} \operatorname{Max}\left(1,\left|\alpha^{(i)}\right|\right), \alpha^{(0)}=\alpha .
$$

For any polynomial $P$ (in any number of variables) whose coefficients are algebraic numbers, we denote by $\operatorname{deg}_{x} P$ the degree of $P$ in the variable $x$, by $H(P)$ the height of $P$, that is, the maximum of the houses of the coefficients of $P$, and by $L(P)$ the length of $P$, that is, the sum of the houses of the coefficients of $P$. For any algebraic number $\alpha$ with minimal defining polynomial $Q$, we put $\operatorname{deg} \alpha=\operatorname{deg} Q, H(\alpha)=H(Q)$ and $L(\alpha)=L(Q)$.

Now we recall the definition of Mahler's $S$-numbers. Let $\omega$ be any complex number. Then we define a function $w_{d}(\omega, h)$ by

$$
\begin{array}{r}
w_{d}(\omega, h)=\operatorname{Min}\{|P(\omega)| ; P(x) \in \mathbb{Z}[x], \operatorname{deg} P \leq d, \\
H(P) \leq h \text { and } P(\omega) \neq 0\} .
\end{array}
$$

Further, we define $w_{d}(\omega)$ and $w(\omega)$ by

$$
w_{d}(\omega)=\limsup _{h \rightarrow \infty} \frac{-\log w_{d}(\omega, h)}{\log h} \text { and } w(\omega)=\limsup _{d \rightarrow \infty} \frac{w_{d}(\omega)}{d} .
$$


Then a number $\omega$ is transcendental if and only if $w(\omega)$ is positive. Then, according to the classification of Mahler, a transcendental number $\omega$ is called an $S$-number if $w(\omega)$ is finite (that is, $w_{d}(\omega) / d$ is bounded as a function of $d)$. For any $S$-number $\omega$, we define the type of $\omega$ by the supremum of the sequence $\left\{w_{d}(\omega) / d\right\}_{d \in \mathbb{N}}$. In this terminology, Galochkin's theorem states that the number $f(\alpha)$ is an $S$-number of type at most $(2 r+1)^{2} b c^{-1} \chi_{0}^{2}$.

In the present paper, we shall prove the following theorems.

THEOREM 1. Let $K$ be an algebraic number field of finite degree. Let $f(z)$ be a function which is transcendental over $\mathbb{C}(z)$ and holomorphic in some neighborhood $U$ of the origin, and satisfies the functional equation (1.1) with $a_{i j}(z) \in K[z]$. Suppose that the coefficients of $f(z)$ in its Taylor series expansion at the origin all lie in the field $K$. Let $\alpha \in U$ be an algebraic number with $0<|\alpha|<1$ such that (1.2) holds for any $k(k=0,1,2, \ldots)$. Put

$$
b=\chi_{1}^{-1} \log M(\alpha), c=\log |\alpha|^{-1} \text { and } \chi_{0}=[K(\alpha): \mathbb{Q}],
$$

where $\chi_{1}$ is the degree of $\alpha$ and $M(\alpha)$ is the Mahler measure of $\alpha$. Then, for any positive integer $d$, we have

$$
w_{d}(f(\alpha)) \leq\left\{r(1+1 / \sqrt{r})^{2} b c^{-1} \chi_{0}^{2}+1\right\} d-1 .
$$

In particular, the number $f(\alpha)$ is an $S$-number of type at most

$$
r(1+1 / \sqrt{r})^{2} b c^{-1} \chi_{0}^{2}+1 .
$$

Corollary. In the above theorem, suppose $K=\mathbb{Q}$ and $\alpha=1 / a$ ( $a \in$ $\mathbb{Z},|a| \geq 2)$. Then, for any positive integer $d$, we have

$$
w_{d}(f(\alpha)) \leq\left\{r(1+1 / \sqrt{r})^{2}+1\right\} d-1 .
$$

In particular, the number $f(\alpha)$ is an $S$-number of type at most $r(1+1 / \sqrt{r})^{2}+$ 1 .

By specializing our situation, we can also give good lower bounds of the values $w_{d}(f(\alpha))$ for small $d$. Namely,we can prove the following theorem.

THEOREM 2. Let $F_{r}(z)$ be the function defined by

$$
F_{r}(z)=\sum_{v=0}^{\infty} z^{r^{v}} \quad(r \in \mathbb{Z}, r \geq 2),
$$

and $a$ be an integer with $|a| \geq 2$. Put

$$
d_{0}=\left\{\begin{array}{l}
(r-2) / 2 \text { if } r \text { is even } \\
(r-1) / 2 \text { if } r \text { is odd }
\end{array}\right.
$$


Then we have

$$
\begin{aligned}
w_{d}\left(F_{r}(1 / a)\right) & =r-1 \text { for } d=1, \ldots, d_{0}, \\
r-1 \leq w_{d}\left(F_{r}(1 / a)\right) & \leq \frac{r d}{r-d} \text { for } d=d_{0}+1, \ldots, r-1,
\end{aligned}
$$

and

$$
w_{d}\left(F_{r}(1 / a)\right) \leq\left\{r(1+1 / \sqrt{r})^{2}+1\right\} d-1 \text { for } d \geq r .
$$

In particular, the number $F_{r}(1 / a)$ is an $S$-number of type at least $r-1$ and at most $r(1+1 / \sqrt{r})^{2}+1$.

REMARK. In the above theorem, we have the equality $w_{1}\left(F_{r}(1 / a)\right)=r-1$ for any $r \geq 3$. But according to a theorem of Shallit [7], the number $F_{r}(1 / a)$ has the continued fraction expansion with bounded partial quotients, and hence we have also the equality $w_{1}\left(F_{2}(1 / a)\right)=1$. We note that the above mentioned equality $w_{1}\left(F_{r}(1 / a)\right)=r-1$ for any $r \geq 3$ is also deduced from a theorem of Shallit [7].

The author would like to express his thanks to Professor Y. Morita for his encouragement. He is also indebted to the referee for his valuable comments.

\section{Preliminaries}

In this section, we give two estimates for $w_{d}(\omega)$ (Lemmas 2 and 3 below). The following lemma is Lemma 5 of Galochkin [2] (cf. also Güting [3, Theorem 6]).

LemMA 1. Let $\alpha_{1}, \ldots, \alpha_{s}$ be algebraic numbers of degrees $\chi_{1}, \ldots, \chi_{s}$. Let $K$ be an algebraic number field, and $I_{K}$ be its integer ring. Put $\chi_{0}=$ $\left[K\left(\alpha_{1}, \ldots, \alpha_{s}\right): \mathbb{Q}\right]$. Let $A\left(x_{1}, \ldots, x_{s}\right) \in I_{K}\left[x_{1}, \ldots, x_{s}\right]$ be a polynomial of $\operatorname{deg}_{x_{i}} A \leq d_{i}$ for each $i$. If $A\left(\alpha_{1}, \ldots, \alpha_{s}\right) \neq 0$, then we have

$$
\left|A\left(\alpha_{1}, \ldots, \alpha_{s}\right)\right| \geq L(A)^{1-\chi_{0}} \prod_{i=1}^{s} M\left(\alpha_{i}\right)^{-\left(\chi_{0} / x_{i}\right) d_{i}} .
$$

REMARK. Checking the proof of Theorem 6 of Güting [3], it is found that we may use $M\left(\alpha_{i}\right)$ in the above inequality instead of $L\left(\alpha_{i}\right)$ which is used by Galochkin [2, Lemma 5] (and also used by Güting [3, Theorem 6]). Note that we have the inequality $M\left(\alpha_{i}\right) \leq L\left(\alpha_{i}\right)$ because of an inequality of Mahler [5].

The following lemma follows from the arguments of Galochkin [2]. 
LEMMA 2. Let $\omega$ be a complex number, and $\alpha$ be an algebraic number of $\operatorname{deg} \alpha=\chi_{1}$ with $0<|\alpha|<1$. Let $\varphi(k)$ be a function on $\mathbb{N}$ such that, for sufficintly large $k \in \mathbb{N}, \varphi(k)$ is a strictly increasing positive valued function tending to infinity, and such that there exists a positive number $\delta$ satisfying

$$
\limsup _{k \rightarrow \infty} \frac{\varphi(k+1)}{\varphi(k)}=\delta<\infty .
$$

Let $K$ be an algebraic number field, and denote by $I_{K}$ its integer ring. Put $\chi_{0}=[K(\alpha): \mathbb{Q}]$. Let $d$ be a positive integer, and $E>1$ be a real number satisfying

$$
\log E>b \chi_{0} d \quad\left(b=\chi_{1}^{-1} \log M(\alpha)\right) .
$$

Suppose that there exists a sequence of polynomials $\left\{R_{k}(z, y)\right\}_{k \in \mathbb{N}}$ such that $R_{k}(z, y) \in I_{K}[z, y]$ and $\operatorname{deg}_{y} R_{k} \leq m$ for any $k$ with a certain positive integer $m$, and such that $R_{k}(z, y)$ satisfies

$$
\begin{gathered}
\log L\left(R_{k}\right)=o(\varphi(k)), \operatorname{deg}_{z}\left(R_{k}\right) \leq \varphi(k)(1+o(1)), \\
\left|R_{k}(\alpha, \omega)\right|=E^{-\varphi(k)(1+o(1))}
\end{gathered}
$$

as $k \rightarrow \infty$. Then we have

$$
w_{d}(\omega) \leq \frac{\delta m \chi_{0} \log E}{\log \left(E / M(\alpha)^{\chi_{0} d / \chi_{1}}\right)}+d-1
$$

Proof. It is convenient for our purpose to work with Koksma's function $w_{d}^{*}$ instead of Mahler's function $w_{d}$. Here we recall the definition (cf. Schneider [6]). For a complex number $\omega$, we define a function $w_{d}^{*}(\omega, h)$ by

$$
w_{d}^{*}(\omega, h)=\operatorname{Min}\{|\omega-\beta| ; \beta \in \overline{\mathbb{Q}}, \operatorname{deg} \beta \leq d, H(\beta) \leq h \text { and } \omega \neq \beta\},
$$

where $\overline{\mathbb{Q}}$ is the field of all algebraic numbers. Then we define $w_{d}^{*}(\omega)$ by

$$
w_{d}^{*}(\omega)=\limsup _{h \rightarrow \infty} \frac{-\log \left(h w_{d}^{*}(\omega, h)\right)}{\log h} .
$$

In what follows, we shall prove

$$
w_{d}^{*}(\omega) \leq \frac{\delta m \chi_{0} \log E}{\log \left(E / M(\alpha)^{\chi_{0} d / \chi_{1}}\right)},
$$

where $\omega$ is a complex number which satisfies all the conditions in the lemma. Since we have $w_{d}(\omega) \leq w_{d}^{*}(\omega)+d-1$ (cf. Schneider [6, Hilfssatz 19]), this proves the lemma.

Let $\beta$ be any algebraic number with $\operatorname{deg} \beta \leq d$ and $H(\beta) \leq h$. Put $\Delta=|\omega-\beta|$. We must give a good lower bound for $\Delta$ which leads to (2.5), 
We may assume $\Delta \leq 1$ without loss of generality. Let $\varepsilon$ be any (small) positive number. Put

$$
R=E^{1-2 \varepsilon} M(\alpha)^{-\left(\chi_{0} d / \chi_{1}\right)(1+\varepsilon)}
$$

We may assume that $R>1$ because of (2.2). Choose a positive integer $k$ such that

$$
R^{\varphi(k-1) / x_{0} m} \leq h<R^{\varphi(k) / x_{0} m} .
$$

By taking a sufficiently large $h$ as an upper bound for $H(\beta)$, we may assume that $k$ is also sufficiently large. We claim that

$$
\Delta \geq E^{-\varphi(k)(1+2 \varepsilon)} .
$$

Indeed, if (2.7) is false, then by (2.3), we have

$$
\begin{aligned}
\left|R_{k}(\alpha, \beta)\right| & \geq\left|R_{k}(\alpha, \omega)\right|-\left|R_{k}(\alpha, \omega)-R_{k}(\alpha, \beta)\right| \\
& \geq E^{-\varphi(k)(1+\varepsilon)}-L\left(R_{k}\right) m(|\omega|+1)^{m} \Delta \\
& \geq E^{-\varphi(k)(1+\varepsilon)}-E^{-\varphi(k)(1+3 \varepsilon / 2)}>0 .
\end{aligned}
$$

Then, by Lemma 1 and (2.3), we have

$$
\begin{aligned}
\left|R_{k}(\alpha, \omega)\right| & \geq\left|R_{k}(\alpha, \beta)\right|-\left|R_{k}(\alpha, \omega)-R_{k}(\alpha, \beta)\right| \\
& \geq L\left(R_{k}\right)^{1-\chi_{0} d} M(\alpha)^{-\left(\chi_{0} d / \chi_{1}\right) \varphi(k)(1+\varepsilon / 2)} M(\beta)^{-\chi_{0} m}-E^{-\varphi(k)(1+3 \varepsilon / 2)} \\
& \geq M(\alpha)^{-\left(\chi_{0} d / \chi_{1}\right) \varphi(k)(1+\varepsilon)} h^{-\chi_{0} m}-E^{-\varphi(k)(1+3 \varepsilon / 2)} .
\end{aligned}
$$

Comparing this lower bound with an upper bound

$$
\left|R_{k}(\alpha, \omega)\right| \leq E^{-\varphi(k)(1-\varepsilon)},
$$

we conclude

$$
h \geq\left(E^{1-2 \varepsilon} M(\alpha)^{-\left(\chi_{0} d / \chi_{1}\right)(1+\varepsilon)}\right)^{\varphi(k) / \chi_{0} m}=R^{\varphi(k) / \chi_{0} m} .
$$

Since this inequality contradicts (2.6), our claim is proved.

Now, by (2.1) and (2.6), we have

$$
\varphi(k) \leq \delta(1+\varepsilon) \varphi(k-1) \leq \frac{(1+\varepsilon) \delta \chi_{0} m \log h}{\log R} .
$$

Hence, by (2.7), we obtain

$$
\Delta \geq h^{-(1+4 \varepsilon) \delta \chi_{0} m(\log E) / \log \left(E^{1-2 e} M(\alpha)^{-\left(\chi_{0} d / \chi_{1}\right)(1+\varepsilon)}\right)} .
$$

Since we can take $\varepsilon$ arbitrarily small, this leads (2.5). The lemma is proved.

We need the following lemma to prove Theorem 2. 
Lemma 3. Let $\omega$ be a real number, $Q>1$ be a real number, and $r \geq 2$ be an integer. Put $E=Q^{r-1}$. Suppose that there exists a sequence of rational numbers $\left\{p_{k} / q_{k}\right\}_{k \in \mathbb{N}}$ such that $p_{k}$ and $q_{k}>0$ are relatively prime integers, and satisfy

$$
q_{k}=Q^{r^{k}(1+o(1))} \text { and }\left|q_{k} \omega-p_{k}\right|=E^{-r^{k}(1+o(1))}
$$

as $k \rightarrow \infty$. Let $d_{0}$ be the number defined by (1.5) in Theorem 2. Then we have

$$
w_{d}(\omega)=r-1 \text { for } d=1, \ldots, d_{0}
$$

and

$$
r-1 \leq w_{d}(\omega) \leq \frac{r d}{r-d} \text { for } d=d_{0}+1, \ldots, r-1 .
$$

This is a special case of Lemma 1 of Amou [1].

\section{Proof of the theorems}

Proof of Theorem 1. Put $\omega=f(\alpha)$. Note that we may assume without loss of generality that $A_{i}(z, y) \in I_{K}[z, y](i=1,2)$. Let $m$ and $n$ be any positive integers. By the theory of homogeneous linear equations, we can construct an auxiliary polynomial $R_{0}(z, y) \in I_{K}[z, y], R_{0}(z, y) \neq 0$, such that

$$
\operatorname{deg}_{z} R_{0} \leq n, \operatorname{deg}_{y} R_{0} \leq m \text { and } \operatorname{ord} R_{0}(z, f(z))>(m+1) n,
$$

where ord $R_{0}(z, f(z))$ is the order of zeros of the function $R_{0}(z, f(z))$ at $z=0$. Since $f(z)$ is transcendental over $\mathbb{C}(z)$, we have $R_{0}(z, f(z)) \neq 0$, and hence we can write ord $R_{0}(z, f(z))=\lambda n(m+1)$ for some $\lambda>1$. Then, because of the functional equation (1.1) for $f(z)$, for any positive integer $k$, we can construct $R_{k}(z, y) \in I_{K}[z, y]$ inductively by taking

$$
R_{k}(z, f(z))=A_{2}(z, f(z))^{m} R_{k-1}(T z, f(T z)) .
$$

We can easily show that

$$
\operatorname{deg}_{z} R_{k} \leq e(k):=\left[n r^{k}(1+\varepsilon(m, n)] \text { and } \operatorname{deg}_{y} R_{k} \leq m,\right.
$$

where $\varepsilon(m, n)$ is a positive valued function of $m, n \in \mathbb{N}$ satisfying $\varepsilon(m, n)$ $\rightarrow 0$ as $m / n \rightarrow 0$. Further, by Lemma 3 of Galochkin [2], we have

$$
L\left(R_{k}\right) \leq(2 L)^{m k} L\left(R_{0}\right) \text { and }\left|R_{k}(\alpha, \omega)\right|=e^{-c \lambda n(m+1) r^{k}(1+o(1))}
$$

as $k \rightarrow \infty$, where $L=\operatorname{Max}\left\{L\left(a_{i j}(z)\right) ; i, j=1,2\right\}$ and $c=\log |\alpha|^{-1}$. 
We fix the following notation. Let $S(m, n)$ be the set of all polynomials $R_{0}(z, y) \in I_{K}[z, y], R_{0}(z, y) \neq 0$, satisfying (3.1). Put

$$
\lambda(m, n)=\sup \left\{\frac{1}{(m+1) n} \text { ord } R_{0}(z, f(z)) ; R_{0}(z, y) \in S(m, n)\right\} .
$$

Let $\lambda(m)$ be the number defined by

$$
\lambda(m)=\limsup _{n \rightarrow \infty} \lambda(m, n) .
$$

This number plays an essential role in our proof.

Put

$$
m=\left[b c^{-1} \chi_{0} d(1+\tau)\right], \quad \tau=1 / \sqrt{r},
$$

where $b, c$ and $\chi_{0}$ are the numbers defined by (1.3). In the following argument, we consider two cases.

CASE I. $\lambda(m)>\sqrt{r}=\tau^{-1}$. Let $\varepsilon$ be any (small) positive number. In this case, there are infinitely many $n$ satisfying $\lambda(m, n)>\tau^{-1}$. We take and fix such an $n$ with $1+\varepsilon(m, n) \leq\left\{\tau\left(\tau^{-1}-\varepsilon\right)\right\}^{-1}$, where $\varepsilon(m, n)$ is the quantity in (3.2). Then we have a sequence of polynomials $R_{k}(z, y) \in I_{K}[z, y]$ for $k \in \mathbb{N}$ satisfying (3.1), (3.2) and (3.3) with $\lambda>\tau^{-1}$. Put

$$
E=e^{c\left(\tau^{-1}-\varepsilon\right)(m+1)} \text { and } \varphi(k)=\lambda\left(\tau^{-1}-\varepsilon\right)^{-1} n r^{k}
$$

for $k \in \mathbb{N}$. Because of our choice of $m$, we may assume that $\log E>b \chi_{0} d$ by taking $\varepsilon$ small enough. Then, all of the conditions in Lemma 2 are satisfied. Put $\gamma=b c^{-1} \chi_{0}$. Since we can take $\varepsilon$ arbitrarily small, applying Lemma 2 to this situation and letting $\varepsilon \rightarrow 0$, we obtain from (2.4) and from our choice of $m$ that

$$
\begin{aligned}
w_{d}(\omega)-d+1 & \leq \frac{r m \chi_{0} c \tau^{-1}(m+1)}{\left\{c \tau^{-1}(m+1)-b \chi_{0} d\right\}} \leq \frac{r(1+\tau) \gamma \chi_{0} d}{1-\frac{\gamma d}{\tau^{-1}(m+1)}} \\
& \leq \frac{r(1+\tau) \gamma \chi_{0} d}{1-\frac{\gamma d}{\tau^{-1} \gamma d(1+\tau)}}=r(1+\tau)^{2} \gamma \chi_{0} d=r(1+1 / \sqrt{r})^{2} b c^{-1} \chi_{0}^{2} d .
\end{aligned}
$$

CASE II. $\lambda(m) \leq \tau^{-1}$. Let $\varepsilon$ be any (small) positive number. We shall construct a finite sequence of positive integers $\left\{n_{i}\right\}_{1 \leq i \leq t}$ which satisfies suitable conditions. First we take a positive integer $n_{1}$ with $n_{1} \geq \tau / \varepsilon$ such that, for any $n \in \mathbb{N}$ with $n \geq n_{1}$, we have

$$
1+\varepsilon(m, n) \leq(1-\varepsilon)^{-1} \text { and } \lambda(m, n) \leq \tau^{-1}(1+2 \varepsilon) /(1+\varepsilon) .
$$

Next we take the least positive integer satisfying $\lambda\left(m, n_{1}\right) n_{1}(1+\varepsilon) \leq n_{2}$. Then we have

$$
1+\varepsilon \leq \frac{\lambda\left(m, n_{2}\right) n_{2}}{\lambda\left(m, n_{1}\right) n_{1}} \leq \tau^{-1}(1+3 \varepsilon)
$$


Further we can take positive integers $n_{3}, n_{4}, \ldots$ such that $n_{i+1}$ is the least positive integer satisfying $\lambda\left(m, n_{i}\right) n_{i}(1+\varepsilon) \leq n_{i+1}(i=2,3, \ldots)$. Thus we obtain a sequence of positive integers $\left\{n_{i}\right\}_{i \in \mathrm{N}}$ satisfying

$$
1+\varepsilon \leq \frac{\lambda\left(m, n_{i+1}\right) n_{i+1}}{\lambda\left(m, n_{i}\right) n_{i}} \leq \tau^{-1}(1+3 \varepsilon)
$$

for any $i \in \mathbb{N}$. Let $t$ be the least positive integer satisfying

$$
\frac{\lambda\left(m, n_{1}\right) n_{1} r}{\lambda\left(m, n_{t}\right) n_{t}} \leq \tau^{-1}(1+3 \varepsilon) .
$$

Note that the left-hand side of the above inequality is greater than 1 . We now have a finite sequence $\left\{n_{i}\right\}_{1 \leq i \leq t}$ which can be used below to define a sequence of polynomials $R_{k}(z, y) \in I_{K}[z, y](k \in \mathbb{N})$ and a function $\varphi(k)$ of $k \in \mathbb{N}$.

For any $i \in \mathbb{N}$ with $1 \leq i \leq t$, we take a polynomial $R_{i, 0}(z, y) \in I_{K}[z, y]$ such that

$$
\operatorname{deg}_{z} R_{i, 0} \leq n_{i}, \operatorname{deg}_{y} R_{i, 0} \leq m
$$

and

$$
\text { ord } R_{i, 0}(z, f(z))=\lambda\left(m, n_{i}\right) n_{i}(m+1) .
$$

Then, for any positive integer $j$, we can construct $R_{i, j}(z, y) \in I_{K}[z, y]$ inductively by taking

$$
R_{i, j}(z, f(z))=A_{2}(z, f(z))^{m} R_{i, j-1}(T z, f(T z)) .
$$

Let us write any $k \in \mathbb{N}$ as $k=j(k) t+i(k)$ where $i(k), j(k)$ are integers with $0 \leq i(k)<t$. In this notation, for any $k \in \mathbb{N}$, we define $R_{k}(z, y)$ and $\varphi(k)$ by

$$
R_{k}(z, y)= \begin{cases}R_{i(k), j(k)}(z, y) & \text { if } i(k) \neq 0 \\ R_{t, j(k)-1}(z, y) & \text { if } i(k)=0\end{cases}
$$

and by

$$
\varphi(k)= \begin{cases}\lambda\left(m, n_{i(k)}\right) n_{i(k)} r^{j(k)}(1-\varepsilon)^{-1} & \text { if } i(k) \neq 0, \\ \lambda\left(m, n_{t}\right) n_{t} r^{j(k)-1}(1-\varepsilon)^{-1} & \text { if } i(k)=0 .\end{cases}
$$

Put $E=e^{c(m+1)(1-\varepsilon)}$. As in Case I, we may assume that $\log E>b \chi_{0} d$ by taking $\varepsilon$ small enough. Then $R_{k}(z, y)(k \in \mathbb{N}), \varphi(k)$ and $E$ satisfy the conditions (2.1) with $\delta \leq \tau^{-1}(1+3 \varepsilon),(2.2)$ and (2.3) in Lemma 2. Since we can take $\varepsilon$ arbitrarily small, applying Lemma 2 to this situation and letting 
$\varepsilon \rightarrow 0$, we obtain from (2.4) and from our choice of $m$ that

$$
\begin{aligned}
w_{d}(\omega)-d+1 & \leq \frac{\tau^{-1} m \chi_{0} c(m+1)}{c(m+1)-b \chi_{0} d} \leq \frac{\tau^{-1}(1+\tau) \gamma \chi_{0} d}{1-\frac{\gamma d}{m+1}} \\
& \leq \frac{\tau^{-1}(1+\tau) \gamma \chi_{0} d}{1-\frac{1}{1+\tau}} \\
& =\tau^{-2}(1+\tau)^{2} \gamma \chi_{0} d=r(1+1 / \sqrt{r})^{2} b c^{-1} \chi_{0}^{2} d .
\end{aligned}
$$

In any case, we obtain $w_{d}(\omega) \leq\left\{r(1+1 / \sqrt{r})^{2} b c^{-1} \chi_{0}^{2}+1\right\} d-1$. This is (1.4), and we have proved the theorem.

If $K=\mathbb{Q}$ and $\alpha=1 / a(a \in \mathbb{Z},|a| \geq 2)$, then we have $b=c$ and $\chi_{0}=1$, and hence the corollary follows.

REMARK. We can easily show as a corollary of the above proof that, for any $\varepsilon>0$, the inequality

$$
w_{d}(f(\alpha)) \leq\left\{(4+\varepsilon) \sqrt{r} b c^{-1} \chi_{0}^{2}+1\right\} d-1
$$

holds for infinitely many $d$.

Proof of Theorem 2. Since the function $f_{r}(z)$ satisfies the functional equation $f_{r}\left(z^{r}\right)=f_{r}(z)-z$, by the corollary of Theorem 1 , we have (1.8). Now, we show (1.6) and (1.7). Put $\omega=f_{r}(1 / a)$. For any $k \in \mathbb{N}$, we define a rational number $p_{k} / q_{k}$ by

$$
\frac{p_{k}}{q_{k}}=\sum_{v=0}^{k} a^{-r^{v}}, q_{k}=a^{r^{k}} .
$$

Then $p_{k}$ and $q_{k}>0$ are relatively prime integers, and satisfy

$$
\left|q_{k} \omega-p_{k}\right|=a^{-(r-1) r^{k}(1+o(1))}
$$

as $k \rightarrow \infty$. Put $Q=a$ and $E=a^{r-1}$. Then, by applying Lemma 3, we deduce (1.6) and (1.7) from (2.8) and (2.9) of Lemma 3 respectively. This completes the proof of the theorem.

About the number $F_{r}(1 / a)$, we conjecture that

$$
w_{d}\left(F_{r}(1 / a)\right)=r-1 \text { for } d=1, \ldots, r-1,
$$

and

$$
w_{d}\left(F_{r}(1 / a)\right)=d \text { for } d \geq r
$$




\section{References}

[1] M. Amou, 'Approximation to certain transcendental decimal fractions by algebraic numbers', J. Number Theory, to appear.

[2] A. I. Galochkin, 'Transcendence measure of values of functions satisfying certain functional equations', Mat. Zametki 27 (1980); English transl. in Math. Notes 27 (1980), 83-88.

[3] R. Güting, 'Approximation of algebraic numbers by algebraic numbers', Michigan Math. J. 8 (1961), 149-159.

[4] K. Mahler, 'Arithmetische Eigenschafter der Lösungen einer Klasse von Funktionalgleichungen', Math. Ann. 101 (1929), 342-366.

[5] K. Mahler, 'An application of Jensen's formula to polynomials', Mathematika 7 (1960), 98-100.

[6] Th. Schneider, Einführung in die transzendenten Zahlen, Springer, Berlin, 1957.

[7] J. O. Shallit, 'Simple continued fractions for some irrational numbers, II', J. Number Theory 14 (1982), 228-231.

Department of Mathematics

Gumma University

Aramaki-cho 4, Maebashi 371

Japan 